This item was submitted to Loughborough's Research Repository by the author.

Items in Figshare are protected by copyright, with all rights reserved, unless otherwise indicated.

\title{
Extended orthogonal space time block codes in wireless relay networks
}

PLEASE CITE THE PUBLISHED VERSION

PUBLISHER

(C) IEEE

VERSION

VoR (Version of Record)

LICENCE

CC BY-NC-ND 4.0

\section{REPOSITORY RECORD}

Alotaibi, Faisal T., and Jonathon Chambers. 2019. "Extended Orthogonal Space Time Block Codes in Wireless Relay Networks”. figshare. https://hdl.handle.net/2134/5843. 
This item was submitted to Loughborough's Institutional Repository (https://dspace.lboro.ac.uk/) by the author and is made available under the following Creative Commons Licence conditions.

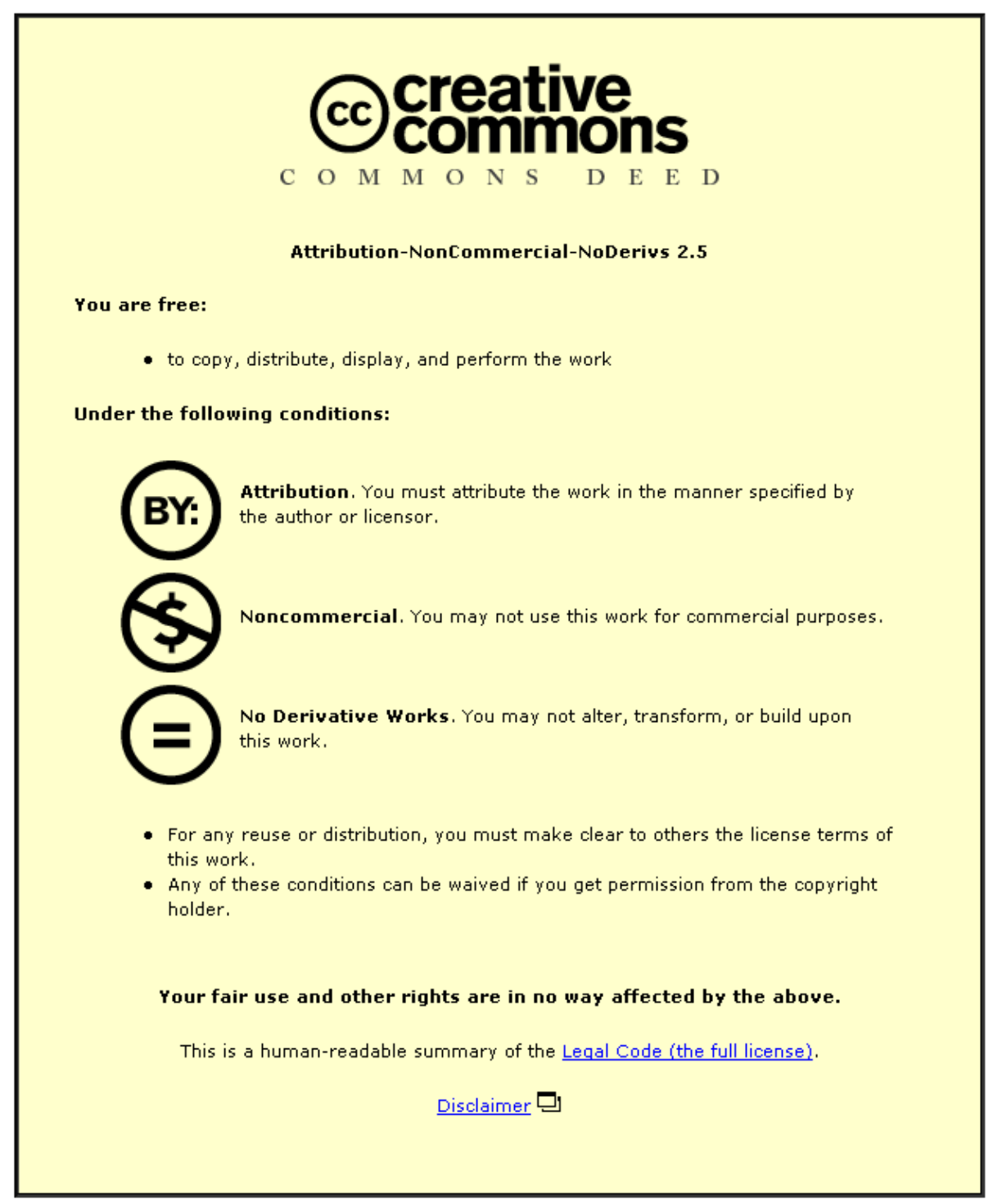

For the full text of this licence, please go to: http://creativecommons.org/licenses/by-nc-nd/2.5/ 
This item was submitted to Loughborough's Institutional Repository (https://dspace.lboro.ac.uk/) by the author and is made available under the following Creative Commons Licence conditions.

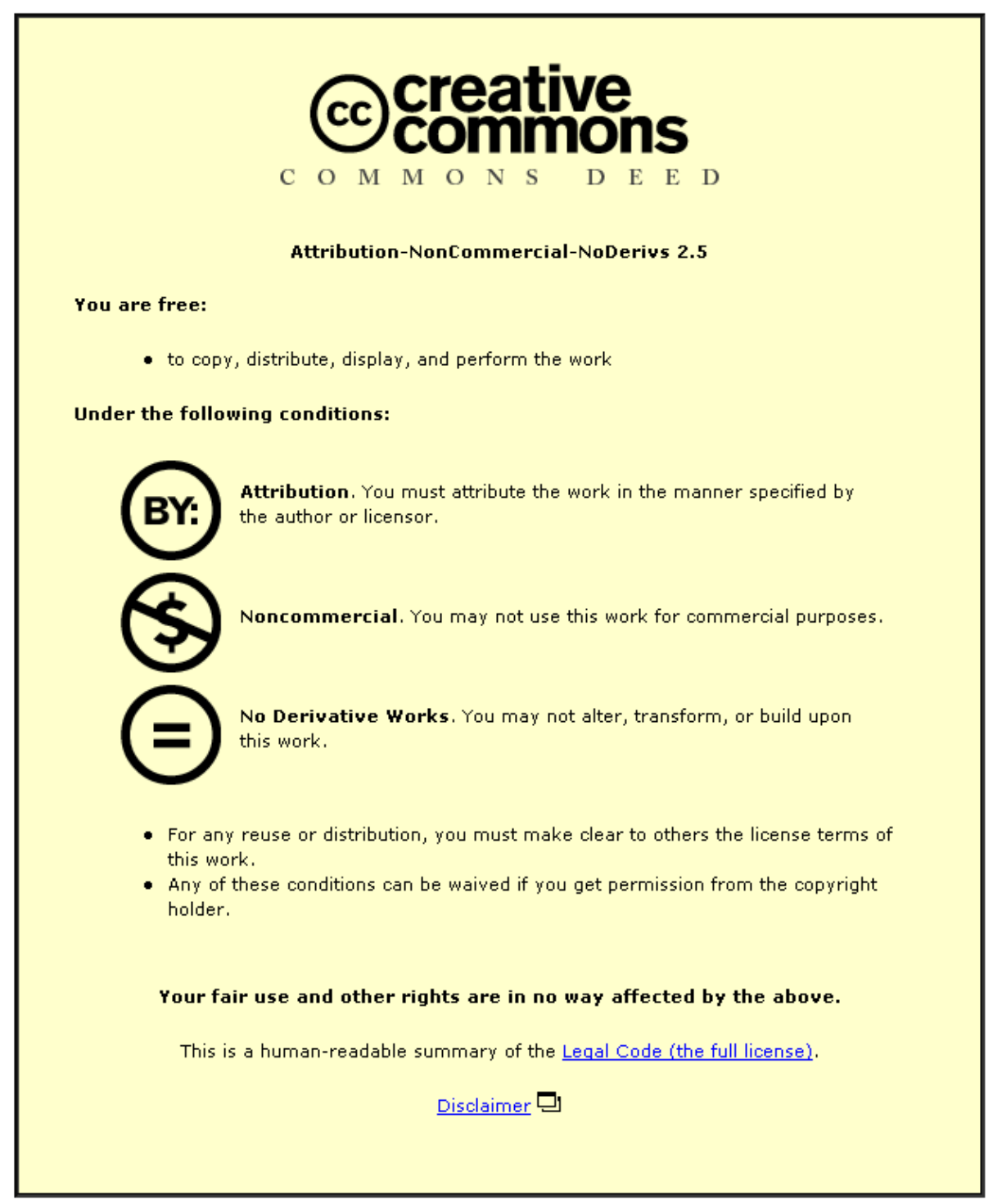

For the full text of this licence, please go to: http://creativecommons.org/licenses/by-nc-nd/2.5/ 


\title{
EXTENDED ORTHOGONAL SPACE TIME BLOCK CODES IN WIRELESS RELAY NETWORKS
}

\author{
F. T. Alotaibi and J. A. Chambers \\ Advanced Signal Processing Group, Department of Electronic and Electrical Engineering \\ Loughborough University, Loughborough LE11 3TU, UK \\ Email: $\{$ F.Alotaibi, J. A. Chambers\}@lboro.ac.uk
}

\begin{abstract}
In this paper we propose complex extended orthogonal space-time block codes (EO-STBCs) with feedback for wireless relay networks with the assumption of quasi-static flat fading channels. Full rate in each stage and full cooperative diversity for distributed EO-STBCs (D-EO-STBCs) are achieved by providing channel state information (CSI) at certain relay nodes. Two closed-loop schemes are proposed which make use of limited feedback from the destination node to a particular number of relay nodes, not exceeding half of the total number of such relay nodes. In our simulations, we use four relay nodes. Simulation results show that these two closed-loop D-EOSTBCs achieve full cooperative diversity in addition to array gain with linear processing. In particular, the proposed D-EO-STBCs designs preserve low decoding complexity and save both transmission power and total transmit time between source and destination.
\end{abstract}

Index Terms - Extended orthogonal space-time block codes (EO-STBCs), orthogonal designs, wireless relay network, cooperative diversity, partial feedback.

\section{INTRODUCTION}

Space-time coding (STC) is an effective technique to exploit spatial diversity not only for multiple-input multiple-output (MIMO) pointto-point systems but also for wireless relay systems [1], thereby improving the reliability, energy usage and throughput. One of the attractive approaches for STC is space-time block codes (STBCs) because of their potential low computational complexity in decoding. They can be divided into two main classes, namely, orthogonal and non-orthogonal, which includes quasi-orthogonal codes [2]. However, orthogonal STBCs are especially promising because full diversity is achieved while a very simple symbolwise maximumlikelihood decoding algorithm can be used at the decoder.

The first real/complex orthogonal space-time block code was proposed by Alamouti [3] for two transmit antennas. In [4] STBCs for more than two transmit antennas from orthogonal designs were proposed. It has been shown in [4] that a complex orthogonal design that provides full diversity and full transmission rate is not possible for more than two antennas. In fact, the Alamouti scheme is the only complex orthogonal STBC that achieves both full transmission rate and full diversity gain. A quasi-orthogonal space-time block code (QO-STBC) is designed in [5] to achieve full transmission rate at the expense of loss in diversity gain and increasing the decoding complexity. Many methods have been proposed for designing STBC schemes with a full rate and full diversity for more than two transmit antennas for example see [6], [7], [8], [9], [10], and [11]. One of these schemes is the extended orthogonal STBC based on closed-loop operation as in [6], which was proposed for three and four transmit antennas, and as in [7], for four transmit antennas. In these two schemes the transmitter exploits channel state information to achieve full transmission rate and full diversity advantage combined with transmit array gain.

In order to exploit the benefits of STBC on transmission energy efficiency and robustness, STBCs were extended into distributed wireless relay networks, which is shown in [1] to be possible by exploiting the cooperating capability of relay nodes. In [12], orthogonal and quasi-orthogonal STBCs, which were originally used in multipleantenna systems, were proposed for distributed STBC in wireless relay networks but without achieving full rate in each stage and full diversity at the same time for more than two relay nodes.

In this paper we extend orthogonal STBCs to wireless relay networks because they can achieve both full rate in each stage and full cooperative diversity gain in addition to array gain. The remainder of the paper is organized as follows. In Section 2, EO-STBCs are described and their extension to relay networks is introduced and analyzed. In Section 3, the simulation results are presented. Finally, conclusions are drawn in Section 4.

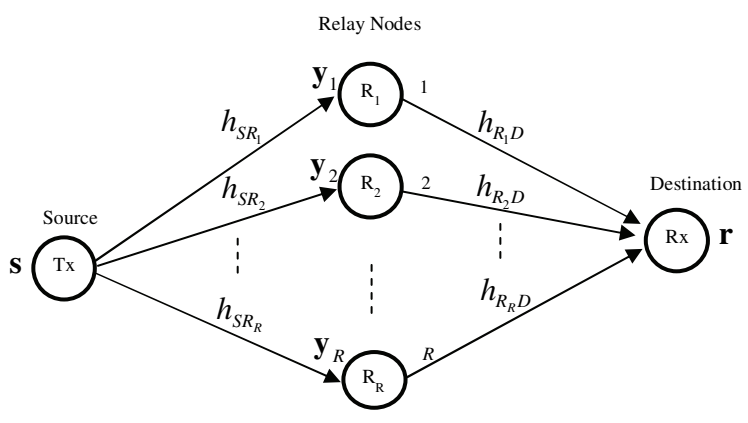

Fig. 1. Wireless relay network with single source and destination together with a relay stage.

\section{EXTENDED ORTHOGONAL STBCS}

Consider a wireless relay system with one source node, one destination node, and $R$ relay nodes, as shown in Fig. 1. Every node in the system has only one antenna. There is no direct link between the transmitter and destination receiver. Denote the fading coefficient from the source node to the $i$ th relay as $h_{S R_{i}}$, and the fading coefficient from the $i$ th relay to the destination node as $h_{R_{i} D}$. Assume the channel between any two terminals is quasi-static flat Rayleigh fading. Therefore, assume that $h_{S R_{i}}$ and $h_{R_{i} D}$ are independent com- 
plex Gaussian random variables with zero-mean and unit-variance. To transmit the information from the source node to the destination node, they undergo two stages. From time 1 to $\mathrm{T}$, which is the first stage, the source node broadcasts the information $\mathbf{s}=\left[s_{1}, \ldots, s_{T}\right]^{T}$ after modulation onto complex symbols to the relay nodes, $R_{i}$, where $(.)^{T}$ denotes vector transpose. The received signal at the $i$ th relay is denoted as $\mathbf{y}_{i}$, which is corrupted by both the fading coefficient $h_{S R_{i}}$ and the noise $\mathbf{n}_{i}$. Meanwhile, the relay nodes $(R)$ receive the information where $R=4$ in this paper. From time $\mathrm{T}+1$ to $2 \mathrm{~T}$, which is the second stage, the source node stops transmission and the relay nodes, which operate in amplify-and-forward (AF) strategy, send the received noisy signals after processing denoted $\mathbf{t}_{i}$ to the destination node. We represent the received signal and noise at the destination receiver by $\mathbf{r}$ and $\mathbf{w}$, respectively. The noises are assumed to be i.i.d. zero-mean and unit-variance complex Gaussian random variables. Clearly

$$
\mathbf{y}_{i}=\sqrt{P_{1} T} h_{S R_{i}} \mathbf{s}+\mathbf{n}_{i} \text { and } \mathbf{r}=\sum_{i=1}^{R} h_{R_{i} D} \mathbf{t}_{i}+\mathbf{w}
$$

where $P_{1}$ denotes the average transmission power at the source node. Then the mean power of the signal $y_{i}$ at a relay node is $P_{1}+1$ due to the unit variance assumption of the additive noise $n_{i}$. Let $P_{2}$ denote the average transmission power at every relay node. The relay nodes will process and transmit the received noisy signals. The transmit signal at the $i$ th relay is designed to be a linear function of its received signal and its conjugate where $(.)^{*}$ denotes the complex conjugation.

$$
\mathbf{t}_{i}=\sqrt{\frac{P_{2}}{P_{1}+1}}\left(A_{i} \mathbf{y}_{i}+B_{i} \mathbf{y}_{i}^{*}\right)
$$

so,

$$
\begin{aligned}
\mathbf{t}_{i}= & \sqrt{\frac{P_{2} P_{1} T}{P_{1}+1}}\left(h_{S R_{i}} A_{i} \mathbf{s}+h_{S R_{i}}^{*} B_{i} \mathbf{s}^{*}\right) \\
& +\sqrt{\frac{P_{2}}{P_{1}+1}}\left(A_{i} \mathbf{n}_{i}+B_{i} \mathbf{n}_{i}^{*}\right)
\end{aligned}
$$

where $A_{i}$ and $B_{i}$ are the matrices used at the $i$ th relays. In our system we have used four relays. These relay nodes are designed to use the following matrices:

$$
\begin{gathered}
A_{1}=A_{2}=\left[\begin{array}{ll}
1 & 0 \\
0 & 1
\end{array}\right]=I_{2}, A_{3}=A_{4}=\left[\begin{array}{ll}
0 & 0 \\
0 & 0
\end{array}\right]=0_{2} \\
B_{1}=B_{2}=\left[\begin{array}{ll}
0 & 0 \\
0 & 0
\end{array}\right]=0_{2}, B_{3}=B_{4}=\left[\begin{array}{ll}
0 & -1 \\
1 & 0
\end{array}\right]
\end{gathered}
$$

The matrix $A_{i}=0_{2}$ means that the $i$ th column of the code matrix contains the conjugate of the information $S$ while the matrix $B_{i}=$ $\mathrm{O}_{2}$ means that the $i$ th column contains the information $S$. The matrix $\mathrm{O}_{2}$ denotes the $2 \times 2$ matrix with all zeros. Define

$$
\begin{aligned}
& \hat{A}_{i}=A_{i}, \hat{h}_{S R_{i}}=h_{S R_{i}}, \hat{\mathbf{n}}_{i}=\mathbf{n}_{i}, \mathbf{s}^{(i)}=\mathbf{s}, \text { if } B_{i}=0 \\
& \hat{A}_{i}=B_{i}, \hat{h}_{S R_{i}}=h_{S R_{i}}^{*}, \hat{\mathbf{n}}_{i}=\mathbf{n}_{i}^{*}, \mathbf{s}^{(i)}=\mathbf{s}^{*}, \text { if } A_{i}=0
\end{aligned}
$$

from (2), we have

$$
\mathbf{t}_{i}=\sqrt{\frac{P_{2} P_{1} T}{P_{1}+1}} \hat{h}_{S R_{i}} \hat{A}_{i} \mathbf{s}^{(i)}+\sqrt{\frac{P_{2}}{P_{1}+1}} \hat{A}_{i} \hat{\mathbf{n}}_{i}
$$

with the second equation in (1), the received signal can be calculated to be

$$
\mathbf{r}=\sqrt{\frac{P_{2} P_{1} T}{P_{1}+1}} S H+W
$$

where

$$
\begin{gathered}
S=\left[\hat{A}_{1} \mathbf{s}^{(1)} \ldots \hat{A}_{R} \mathbf{s}^{(R)}\right] \\
H=\left[\hat{h_{S R_{1}} h_{R_{i} D} \ldots \hat{h}_{S R_{R}} h_{R_{R} D}}\right]^{T} \\
W=\sqrt{\frac{P_{2}}{P_{1}+1} \sum_{i=0}^{R} h_{R_{i} D} \hat{A}_{i} \hat{\mathbf{n}_{i}}+\mathbf{w}}
\end{gathered}
$$

Therefore, without decoding, the relays generate a space-time codeword $S$ distributively at the receiver. $H$ is the equivalent channel and $\mathrm{W}$ is the equivalent noise. If $H$ is known at the receiver, the maximum-likelihood (ML) decoding is

$$
\arg \min _{\mathbf{s}}\left\|X-\sqrt{\frac{P_{2} P_{1} T}{P_{1}+1}} S H\right\|_{F}
$$

where $\|\cdot\|_{F}$ indicates the Frobenius norm.

We adopt the optimum power allocation in [13] in our proposed scheme as next described. Denote $P$ as the total transmission power in the whole scheme, so $P_{1}=\frac{P}{2}$ and $P_{2}=\frac{P}{2 R}$ where $R$ is the number of the relay nodes.

\subsection{Open-Loop D-EO-STBCs}

Initially, we assume there is no channel information at the relays but full channel information at the receiver. First, we define the form of the D-EO-STBC code word $S$ that we want to generate at the destination receiver. It has the following form [6]

$$
S=\left[\begin{array}{cccc}
s_{1} & s_{1} & s_{2} & s_{2} \\
-s_{2}^{*} & -s_{2}^{*} & s_{1}^{*} & s_{1}^{*}
\end{array}\right]
$$

so, we defined the information sequence which is encoded at the source node as $\mathbf{s}=\left[s_{1}-s_{2}^{*}\right]^{T}$, as in [12]. It is clear that the extended code word we select, as in [6], is built from the well known Alamouti code. Also, the extended code word has scale-free property [6].

In the case of four relays, the received signal, $\mathbf{r}$, will be written as $\mathbf{r}=\sum_{i=1}^{4} h_{R_{i} D} \mathbf{t}_{i}+\mathbf{w}$ and since the coherence interval $\mathrm{T}=2$, therefore

$$
\mathbf{r}=\left[\begin{array}{ll}
r_{1} & r_{2}
\end{array}\right]^{T}, \mathbf{t}_{i}=\left[\begin{array}{ll}
t_{i 1} & t_{i 2}
\end{array}\right]^{T} \text { and } \mathbf{w}=\left[\begin{array}{ll}
w_{1} & w_{2}
\end{array}\right]^{T}
$$

so, the received signals $r_{1}$ and $r_{2}$ at the two independent time slots are expressed as follows

$$
\begin{aligned}
& r_{1}=t_{11} h_{R_{1} D}+t_{21} h_{R_{2} D}+t_{31} h_{R_{3} D}+t_{41} h_{R_{4} D}+w_{1} \\
& r_{2}=t_{12} h_{R_{1} D}+t_{22} h_{R_{2} D}+t_{32} h_{R_{3} D}+t_{42} h_{R_{4} D}+w_{2}
\end{aligned}
$$

By substituting $\mathbf{t}_{i}$ and taking the conjugate of $r_{2}$, the equivalent channel matrix corresponding to the code word in (4) used over four relay nodes is given by:

$H=\left[\begin{array}{ll}h_{S R_{1}} h_{R_{1} D}+h_{S R_{2}} h_{R_{2} D} & h_{S R_{3}}^{*} h_{R_{3} D}+h_{S R_{4}}^{*} h_{R_{4} D} \\ h_{S R_{3}} h_{R_{3} D}^{*}+h_{S R_{4}} h_{R_{4} D}^{*} & -h_{S R_{1}}^{*} h_{R_{1} D}^{*}-h_{S R_{2}}^{*} h_{R_{2} D}^{*}\end{array}\right]$

Applying the matched filtering at the destination receiver with the equivalent channel matrix in (8), we can obtain the Grammian matrix $\mathrm{G}$ as follows:

$$
G=H^{H} H=\left[\begin{array}{cc}
\alpha & 0 \\
0 & \alpha
\end{array}\right]
$$

where $(.)^{H}$ denotes Hermitian transpose and $\alpha$ represents the channel gain such that

$$
\alpha=\sum_{i=1}^{4}\left|h_{S R_{i}} h_{R_{i} D}\right|^{2}+\beta_{1}+\beta_{2}
$$


with

$$
\begin{aligned}
\beta_{1} & =h_{S R_{1}}^{*} h_{R_{1} D}^{*} h_{S R_{2}} h_{R_{2} D}+h_{S R_{2}}^{*} h_{R_{2} D}^{*} h_{S R_{1}} h_{R_{1} D} \\
& =2 \operatorname{Re}\left\{h_{S R_{2}}^{*} h_{R_{2} D}^{*} h_{S R_{1}} h_{R_{1} D}\right\} \\
\beta_{2} & =h_{S R_{3}}^{*} h_{R_{3} D} h_{S R_{4}} h_{R_{4} D}^{*}+h_{S R_{4}}^{*} h_{R_{4} D} h_{S R_{3}} h_{R_{3} D}^{*} \\
& =2 \operatorname{Re}\left\{h_{S R_{3}}^{*} h_{R_{3} D} h_{S R_{4}} h_{R_{4} D}^{*}\right\}
\end{aligned}
$$

where $\beta_{1}, \beta_{2}$ are the interference factors and $|.|^{2}$ denotes the modulus squared of a complex number and $R e\{$.$\} its real part.$

From (6), it is evident that the Grammian matrix of the D-EO-STBC is orthogonal, which indicates that the code can be decoded with a simple receiver. It can be shown that the signal-to-noise ratio (SNR) is

$$
S N R=\alpha \frac{\sigma_{x}^{2}}{\sigma_{n}^{2}}=\left(\sum_{i=1}^{4}\left|h_{S R_{i}} h_{R_{i} D}\right|^{2}+\beta_{1}+\beta_{2}\right) \frac{\sigma_{x}^{2}}{\sigma_{n}^{2}}
$$

where $\sigma_{x}^{2}$ is the total transmit power of the desired signal, and $\sigma_{n}^{2}$ is the noise power at the receiver.

It can be seen that the $\beta_{1}$ and $\beta_{2}$ terms may reduce channel gain, and correspondingly the SNR. In order to achieve a full cooperative diversity of order four for a wireless system with four relays, we used two feedback schemes.

\subsection{Closed-Loop D-EO-STBCs for Four Relay Nodes}

We now consider there is channel information at the relays. In our four relay system, partial channel information is fed into only any two relays each having different code processing which are enough to leverage the system gain to the maximum as shown in [6] and [7]. The two relays that we selected in our system are $R_{1}$ and $R_{3}$ as shown in Fig. 2. We assumed that the full channel information CSI can be estimated at the destination receiver. So, the relay transmitted signals $\mathbf{t}_{1}$ and $\mathbf{t}_{3}$ are multiplied by $U_{1}$ and $U_{2}$ before they are transmitted from the first and third relay nodes, respectively, while the other two are kept unchanged; we used in our simulation two feedback schemes as in [6] and [7] to determine the value of $U_{1}$ and $U_{2}$. In [6], $U_{1}=(-1)^{i}$ and $U_{2}=(-1)^{k}$, where $\mathrm{i}, \mathrm{k}=0,1$, which means using 0 or $\pi$ as the rotation angle for the signals. In [7], $U_{1}=e^{j \theta_{1}}$ and $U_{2}=e^{j \theta_{2}}$ respectively, which means using the exact angles for rotating the signals which result in maximizing $\beta_{1}$ and $\beta_{2}$. Therefore, the error performance is improved at the expense of increased feedback overhead. The phase rotation on transmitted symbols is equivalent to rotating the phases of the corresponding channel coefficients. The received signals $r_{1}$ and $r_{2}$ at two independent time intervals are expressed as follows

$$
\begin{aligned}
& r_{1}=t_{11} U_{1} h_{R_{1} D}+t_{21} h_{R_{2} D}+t_{31} U_{2} h_{R_{3} D}+t_{41} h_{R_{4} D}+w_{1} \\
& r_{2}=t_{12} U_{1} h_{R_{1} D}+t_{22} h_{R_{2} D}+t_{32} U_{2} h_{R_{3} D}+t_{42} h_{R_{4} D}+w_{2}
\end{aligned}
$$

Using the same procedures as we have followed in the open loop scheme, the equivalent channel matrix $H_{c}$ given by

$$
H_{c}=\left[\begin{array}{cc}
f_{1} & f_{2} \\
f_{2}^{*} & -f_{1}^{*}
\end{array}\right]
$$

where

$$
\begin{aligned}
& f_{1}=U_{1} h_{S R_{1}} h_{R_{1} D}+h_{S R_{2}} h_{R_{2} D} \\
& f_{2}=U_{2} h_{S R_{3}}^{*} h_{R_{3} D}+h_{S R_{4}}^{*} h_{R_{4} D}
\end{aligned}
$$

also,

$$
G=H_{c}^{H} H_{c}=\left[\begin{array}{cc}
\alpha & 0 \\
0 & \alpha
\end{array}\right]
$$

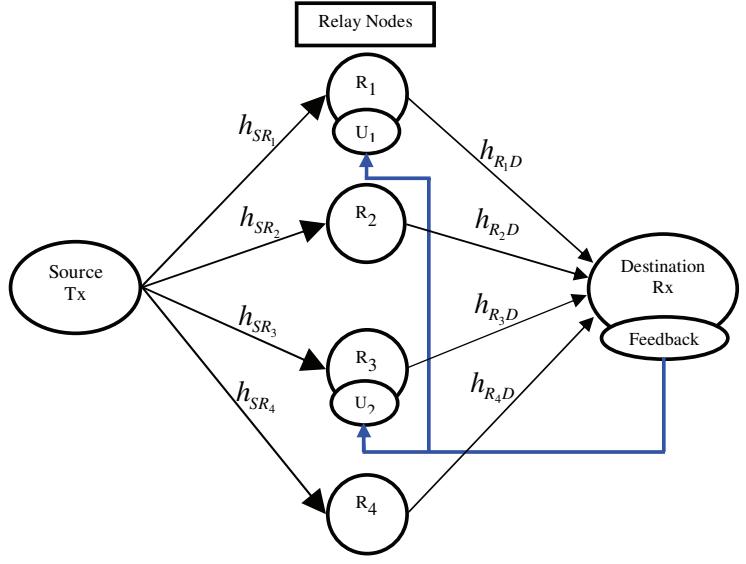

Fig. 2. Schematic representation of the proposed closed-loop D-EOSTBC system for four relay nodes with feedback to two nodes.

with

$$
\alpha=\sum_{i=1}^{4}\left|h_{S R_{i}} h_{R_{i} D}\right|^{2}+\beta_{1}+\beta_{2}
$$

where

$$
\begin{aligned}
& \beta_{1}=2 \operatorname{Re}\left\{U_{1} h_{S R_{2}}^{*} h_{R_{2} D}^{*} h_{S R_{1}} h_{R_{1} D}\right\} \\
& \beta_{2}=2 \operatorname{Re}\left\{U_{2} h_{S R_{3}}^{*} h_{R_{3} D} h_{S R_{4}} h_{R_{4} D}^{*}\right\}
\end{aligned}
$$

taking into account that $\left|U_{1}\right|^{2}=\left|U_{2}\right|^{2}=1$.

The feedback performance gain is $g_{f}=\beta_{1}+\beta_{2}$. From this, it is clear that if $g_{f}>0$, the designed closed-loop system can obtain additional performance gain, which leads to an improved whole channel gain, and correspondingly the $S N R$ at the destination receiver.

According to the analysis in [6], $U_{1}=(-1)^{i}$ and $U_{2}=(-1)^{k}$. So, we can propose the following design criteria:

$$
\begin{aligned}
& i= \begin{cases}0 & \text { if } h_{S R_{2}}^{*} h_{R_{2} D}^{*} h_{S R_{1}} h_{R_{1} D} \geq 0 \\
1 & \text { otherwise }\end{cases} \\
& k= \begin{cases}0 & \text { if } h_{S R_{3}}^{*} h_{R_{3} D} h_{S R_{4}} h_{R_{4} D}^{*} \geq 0 \\
1 & \text { otherwise }\end{cases}
\end{aligned}
$$

While according to the analysis in [7], $U_{1}=e^{j \theta_{1}}$ and $U_{2}=e^{j \theta_{2}}$. So, we can propose the following design criteria:

$$
\begin{aligned}
& \theta_{1}=-\operatorname{angle}\left(h_{S R_{2}}^{*} h_{R_{2} D}^{*} h_{S R_{1}} h_{R_{1} D}\right) \\
& \theta_{2}=-\operatorname{angle}\left(h_{S R_{3}}^{*} h_{R_{3} D} h_{S R_{4}} h_{R_{4} D}^{*}\right)
\end{aligned}
$$

Similarly, we can design a closed-loop scheme for three relay nodes with one feedback link only.

\section{SIMULATION RESULTS}

In this section, we compare the bit error performance of the proposed extended schemes with earlier proposed schemes in quasi-static flat fading channels. The fading is constant within a frame and changes independently from frame-to-frame. Each frame consists of 64 symbols in our simulation. Only one antenna at the transmitter and the receiver was considered, but this technique can be extended to multiple transmit or receive antennas or both which result in better improvement in the performance. All schemes use QPSK modulation and have the same total power. The $\mathrm{x}$-axis shows the average transmitted power from the source node in $\mathrm{dB}$ and the $\mathrm{y}$-axis shows the 


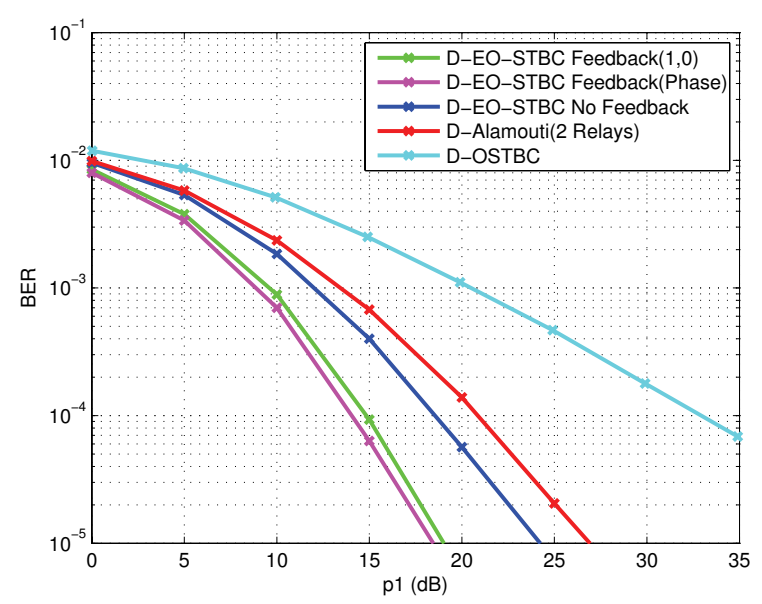

Fig. 3. Performance comparison for various STBCs in a four relay wireless network.

BER.

In Fig. 3, we show the performance of the proposed D-EO-STBC for four relay nodes. We can see that the two closed-loop D-EO-STBCs are better than the D-EO-STBC without feedback. In particular, at a bit error probability of $10^{-4}$, the proposed D-EO-STBC scheme with feedback 0 or 1 provides approximately $3.5 \mathrm{~dB}$ improvement while the proposed scheme with feedback of exact phase provides approximately $4.5 \mathrm{~dB}$ improvement. However, the improvement increases as the average transmitted power increases. This improvement of the two closed loop schemes is because both achieve full cooperative diversity of order four combined with array gain. As shown, the closed loop scheme with exact phase as feedback provides better performance ( approximately $1 \mathrm{~dB}$ for BER of $10^{-4}$ ) compared with the other closed loop scheme with 0 or 1 as a feedback. The figure also provides a comparison of the proposed schemes with the distributed orthogonal STBC (D-OSTBC) [4], using the optimal power allocation protocol for distributed STC with unequal time intervals as in [12], and the distributed Alamouti (D-Alamouti) scheme [3].We clearly notice that the proposed scheme outperforms them. Specifically, when the BER is $10^{-4}$, our open loop scheme can achieve more than $2 \mathrm{~dB}$ gain over the D-Alamouti scheme and more than $14 \mathrm{~dB}$ gain over D-OSTBC.

As shown from the simulation, the exact phase feedback scheme presents the best performance compared to the others. The advantage of the exact phase feedback scheme can be retained by exploiting some quantization of the feedback coefficients as in [7], to yield a more practical scheme with limited feedback. In [7], very little degradation in BER performance is shown so it is not repeated in our simulations.

\section{CONCLUSIONS}

In this paper, we proposed complex extended orthogonal STBCs for wireless relay networks. Simulations showed that D-EO-STBC provides better performance than the D-Alamouti scheme and the DOSTBC when we used both closed loop and open loop schemes. In terms of data rate, the open-loop D-EO-STBC provides full rate in each stage which is equal to the data rate of the D-Alamouti scheme and outperforms the D-OSTBC. These results are very encouraging since the total transmission power is the same or constant in addition to the information symbols being decoded separately in a very simple manner at the receiver. Our simulations also showed that with partial channel knowledge at the relays, the D-EO-STBC achieves higher gain compared with no feedback because the closed-loop DEO-STBC achieves full diversity gain in addition to array gain. The main result we can conclude is that the extended schemes in wireless relay networks save the transmission time and the transmission power. Finally, results in this paper can be extended straightforwardly to wireless relay networks with multiple-antenna nodes.

\section{REFERENCES}

[1] J. N. Laneman and G. W. Wornell, "Distributed space-timecoded protocols for exploiting cooperative diversity in wireless networks," IEEE Trans. on Information Theory, vol. 49, pp. 2415-2425, October 2003.

[2] B. Badic, Space-time block coding for multiple antenna systems, PhD Thesis, Vienna University of Technology, Austria, Nov. 2005.

[3] S. M. Alamouti, "A simple transmit diversity technique for wireless communications," IEEE J.Select.Areas Commun., vol. 16, pp. 1451 - 1458, October 1998.

[4] V. Tarokh, H. Jafarkhani, and A. R. Calderbank, "Space-time block codes from orthogonal designs," IEEE Trans. on Information Theory, vol. 45, pp. 1456-1467, July 1999.

[5] H. Jafarkhani, "A quasi-orthogonal space-time block code," IEEE Trans. Commun., vol. 49, pp. 1-4, Jan 2001.

[6] Y. Yu, S. Keroueden, and J. Yuan, "Closed-loop extended orthogonal space-time block codes for three and four transmit antennas," IEEE Signal Processing letters, vol. 13, pp. 273276, May 2006.

[7] N. M. Eltayeb, S. Lambotharan, and J. A. Chambers, "A phase feedback based extended space-time block code for enhancement of diversity," VTC2007-Spring. IEEE 65th, pp. 22962299, April 2007.

[8] J. Akhtar and D. Gesbert, "Extended orthogonal block codes with partial feedback," IEEE Trans. Wireless Commun., vol. 3, pp. 1959-1962, Nov 2004.

[9] C. Toker, S. Lambotharan, and J. A. Chambers, "Closed-loop quasi-orthogonal STBCs and their performance in multipath fading environments and when combined with turbo codes," IEEE Trans. Wireless Commun., vol. 3, pp. 1890-1896, Nov 2004.

[10] C. Toker, S. Lambotharan, and J. A. Chambers, "Space-time block coding for four transmit antennas with closed loop feedback over frequency selective fading channel," IEEE Information Theory Workshop, Paris, France,March/April 2003.

[11] W. Su and X. G. Xia, "Signal constellations for quasiorthogonal space-time block codes with full diversity," IEEE Trans. on Information Theory, vol. 50, pp. 2331-2347, Oct 2004.

[12] Y. Jing and H. Jafarkhani, "Using orthogonal and quasiorthogonal designs in wireless relay networks," IEEE Trans. on Information Theory, vol. 53, pp. 4106-4118, Nov 2007.

[13] Y. Jing and B. Hassibi, "Distributed space-time coding in wireless relay networks," IEEE Trans. Wireless Commun., vol. 5, pp. 3524-3536, Dec 2006. 